\title{
Pre-existing liver disease is associated with poor outcome in patients with SARS CoV2 infection; The APCOLIS Study (APASL COVID-19 Liver Injury Spectrum Study)
}

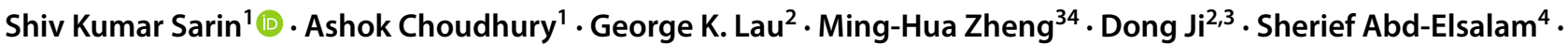 \\ Jaeseok Hwang ${ }^{5}$. Xiaolong $\mathrm{Qi}^{6}$. lan Homer Cua ${ }^{7}$. Jeong III Suh ${ }^{8}$. Jun Gi Park ${ }^{8}$. Opass Putcharoen ${ }^{9}$. \\ Apichat Kaewdech ${ }^{10}$. Teerha Piratvisuth $^{10}$. Sombat Treeprasertsuk ${ }^{9} \cdot$ Sooyoung Park $^{11} \cdot$ Salisa Wejnaruemarn ${ }^{9}$. \\ Diana A. Payawal ${ }^{12}$. Oidov Baatarkhuu ${ }^{13}$. Sang Hoon $\mathrm{Ahn}^{14}$. Chang Dong Yeo ${ }^{14}$. Uzziel Romar Alonzo ${ }^{12}$. \\ Tserendorj Chinbayar ${ }^{15}$. Imelda M. Loho ${ }^{16}$. Osamu Yokosuka ${ }^{17}$. Wasim Jafri ${ }^{18}$. Soeksiam Tan $^{19}$. Lau Ing Soo ${ }^{19}$. \\ Tawesak Tanwandee $^{20} \cdot$ Rino Gani $^{21} \cdot$ Lovkesh Anand $^{22}$. Eslam Saber Esmail ${ }^{4} \cdot$ Mai Khalaf $^{4}$. Shahinul Alam ${ }^{23}$. \\ Chun-Yu Lin ${ }^{24}$. Wan-Long Chuang ${ }^{24} \cdot$ A. S. Soin ${ }^{25}$. Hitendra K. Garg ${ }^{26} \cdot$ Kemal Kalista ${ }^{27}$. Badamnachin Batsukh ${ }^{13}$. \\ Hery Djagat Purnomo ${ }^{28}$. Vijay Pal Dara ${ }^{29}$. Pravin Rathi ${ }^{30} \cdot$ Mamun Al Mahtab $^{23}$. Akash Shukla ${ }^{31} \cdot$ Manoj K. Sharma $^{1}$. \\ Masao Omata ${ }^{32,33}$. APASL COVID Task Force, APASL COVID Liver Injury Spectrum Study (APCOLIS Study-NCT \\ 04345640)
}

Received: 12 June 2020 / Accepted: 27 June 2020 / Published online: 4 July 2020

(c) Asian Pacific Association for the Study of the Liver 2020

\begin{abstract}
Background and aims COVID-19 is a dominant pulmonary disease, with multisystem involvement, depending upon comorbidities. Its profile in patients with pre-existing chronic liver disease (CLD) is largely unknown. We studied the liver injury patterns of SARS-Cov-2 in CLD patients, with or without cirrhosis.

Methods Data was collected from 13 Asian countries on patients with CLD, known or newly diagnosed, with confirmed COVID-19.

Results Altogether, 228 patients [185 CLD without cirrhosis and 43 with cirrhosis] were enrolled, with comorbidities in nearly $80 \%$. Metabolism associated fatty liver disease $(113,61 \%)$ and viral etiology $(26,60 \%)$ were common. In CLD without cirrhosis, diabetes [57.7\% vs 39.7\%, OR $=2.1(1.1-3.7), \mathrm{p}=0.01]$ and in cirrhotics, obesity, [64.3\% vs. $17.2 \%, \mathrm{OR}=8.1$ $(1.9-38.8), \mathrm{p}=0.002]$ predisposed more to liver injury than those without these. Forty three percent of CLD without cirrhosis presented as acute liver injury and $20 \%$ cirrhotics presented with either acute-on-chronic liver failure [5 (11.6\%)] or acute decompensation [4 (9\%)]. Liver related complications increased $(\mathrm{p}<0.05)$ with stage of liver disease; a Child-Turcotte Pugh score of 9 or more at presentation predicted high mortality [AUROC 0.94, HR =19.2 (95 CI 2.3-163.3), $\mathrm{p}<0.001$, sensitivity $85.7 \%$ and specificity $94.4 \%$ ). In decompensated cirrhotics, the liver injury was progressive in $57 \%$ patients, with $43 \%$ mortality. Rising bilirubin and AST/ALT ratio predicted mortality among cirrhosis patients.

Conclusions SARS-Cov-2 infection causes significant liver injury in CLD patients, decompensating one fifth of cirrhosis, and worsening the clinical status of the already decompensated. The CLD patients with diabetes and obesity are more vulnerable and should be closely monitored.
\end{abstract}

Keywords COVID-19 $\cdot$ SARS CoV2 $\cdot$ Acute liver injury $\cdot$ Chronic liver disease

\begin{tabular}{|c|c|c|}
\hline \multirow{4}{*}{$\begin{array}{l}\text { Electronic supplementary material The online version of this } \\
\text { article (https://doi.org/10.1007/s12072-020-10072-8) contains } \\
\text { supplementary material, which is available to authorized users. }\end{array}$} & \multicolumn{2}{|c|}{ Abbreviations } \\
\hline & AARC & $\begin{array}{l}\text { APASL ACLF Research Consortium } \\
\text { (AARC) }\end{array}$ \\
\hline & ACLF & Acute on Chronic Liver Failure \\
\hline & $\mathrm{AD}$ & Acute Decompensation \\
\hline Shiv Kumar Sarin & AKI & Acute Kidney Injury \\
\hline shivsarin@gmail.com & ALI & Acute Liver Injury \\
\hline Extended author information available on the last page of the article & COVID-19 & Coronavirus Disease 2019 \\
\hline
\end{tabular}


SARS CoV2 Severe Acute Respiratory Syndrome Coronavirus 2

CAIDs Cirrhosis Associated Immunue Dysfunction syndrome

CLD Chronic Liver Disease

AST Aspartate Aminotransferase

ALT Alanine Aminotransferase (ALT)

SAP Serum Alkaline Phosphatase

ACE-2 Angiotensin Converting Enzyme-2

MAFLD Metabolic Associated Fatty Liver Disease

\section{Introduction}

The pandemic of respiratory infection with Severe Acute Respiratory Syndrome Corona Virus -2 (SARS-CoV-2) has already infected 7 million people globally, leaving 4 lacs dead [1]. SARS-Cov-2 is an enveloped, non-segmented, positive-sense RNA virus belonging to $\beta$-coronavirus family [2] and causes fever, dry cough and breathing difficulty, which can progress to respiratory distress due to interstitial pneumonia and multi-organ involvement $[3,4]$. The latter is due to systemic inflammation leading to a cytokine storm and immune dysfunction often with features of macrophage activation syndrome, as evidenced by hyperferritinaemia, hepatic dysfunction and diffuse intravascular coagulation [5]. Viral entry is through the ACE-2 receptor; high expression of which is noted in type II alveolar cells, enterocytes, cholangiocytes, myocardial cells and proximal tubule cells of kidney, predisposing the concerned organs to the risk of developing complications [6, 7].

Deranged liver functions, mainly raised alanine aminotransferase (ALT) and aspartate aminotransferase (AST), have been reported in 14-53\% patients without known liver disease $[8,9]$. Patients with severe disease showed higher frequency and degree of liver dysfunction while in milder cases, the liver injury was transient [10]. The mechanisms of hepatic injury include immune-mediated inflammation, hypoxic injury due to severe pneumonia and drug related [11]. It is also postulated that expression of ACE2 receptor on cholangiocytes may predispose to cholestatic injury [12]. Data on post-mortem liver biopsies is limited and demonstrates moderate microvascular steatosis and mild lobular and portal activity [13].

The acute insult in COVID-19 is systemic and it may progress to involve other systems. Comorbidities like diabetes, hypertension, obesity, coronary artery disease and chronic liver disease (CLD) co-exist in general population, more so in middle aged and elderly $[8,14]$. While the true burden of liver disease is not known; these accounted for $4.6 \%$ of all deaths [15]. In fact, liver diseases contribute global disease burden in the form of metabolism associated liver disease (MAFLD), alcohol-associated liver disease and viral hepatitis [16]. Hence, during COVID-19 pandemic, it is very likely that CLD patients would be exposed to SARS-CoV-2 infection. Moreover, many cirrhotic patients are required to attend hospitals regularly and thus become susceptible to SARS-CoV-2 infection. Importantly, the SARS-CoV-2 infection produces lymphocytopenia with or without leukopenia, thrombocytopenia and raised fibrinogen degradation products $[5,8,17]$, which pre-exist in CLD patients due to bone marrow suppression and cirrhosis associated immune dysfunction syndrome (CAIDS) [18].

Acute liver injury in healthy individuals has been reported in a few studies. However, the impact of SARS-Cov-2 infection on pre-existing CLD, compensated and decompensated cirrhosis is largely unknown. While in the presence of normal liver synthetic function, rise in liver enzymes with or without jaundice may be transitory and recover spontaneously. However, whether SARS-Cov-2 infection can inflict serious and prolonged liver injury in patients of CLD and may aggravate already compromised hepatic synthetic functions leading to development of acute-on-chronic liver failure (ACLF), acute decompensation (AD) in a cirrhotic liver or, worsening of prior decompensated liver disease is not known [19]. The severity and incidence of kidney injury, secondary infections, hepatic encephalopathy, gastrointestinal bleeding and mortality, also needs to be known in a large-cohort. The Asia Pacific Association for the Study of the Liver (APASL) launched a pan-Asia collaborative study "APASL COVID Liver Injury Spectrum (APCOLIS, clinical trial identifier NCT04345640). The aim is to study the spectrum of liver injury, complications of liver disease and COVID-19 related complications in relation to pre-existing liver disease and its spectrum with SARS CoV2 infection.

\section{Patients and methods}

\section{Study design}

Data was collected on a web-based performa of COVID-19 patients, seen in Asia, between January to April 2020, under the APASL COVID-19 task force with the help of APASL ACLF Research Consortium (AARC) a multinational registry. Institutional ethics committee approval was obtained [NCT04345640]. All authors had access to the study data, and had the opportunity to review and approve the final manuscript.

\section{Patients}

Admitted patients with COVID-19, 18 years or above, were considered for data source. Patients with known or newly diagnosed CLD or cirrhosis and infected with SARS-Cov-2 were included in the analysis. The presenting 
complaints, laboratory parameters, clinical events and survival outcome till day 28 were obtained. The primary objective was to determine the clinical presentation, biochemical alterations, complications and survival outcome of SARS-Cov-2 infection in the whole spectrum of CLD. The secondary objectives were to compare the pattern of liver injury in relation to existing synthetic functions, COVID-19 disease severity and influence of comorbidities. We also analyzed the predictors of severity of liver injury and influence of etiology on outcomes. The primary end point was death or complete recovery from COVID19. Secondary end points were severity of disease, liver injury profile, complications related to liver disease and in relation to COVID-19.

Diagnosis of COVID-19 Confirmation of SARS-CoV-2 infection was achieved as per guidelines [20], with proper extraction of nucleic acids from the respiratory sample followed by RT-PCR assay for virus detection.

Treatment Individualized antiviral or drug therapy protocol given at respective centres was collected. Generally, isolation for asymptomatic cases, hydroxychloroquine with azithromycin and antiviral drugs (oseltamivir, remdesivir, favipravir, lopinavir + ritonavir) at admission in mild and moderate cases was practiced. The moderate and severe cases received antibiotics, convalescent plasma, steroid in form of intravenous methylprednisolone or IVIG, in case to case basis. Decisions included fluid management, vasopressors, high flow nasal cannula (HNFC) at 10L, and noninvasive or invasive ventilation as per standard protocols.

The liver specific treatment was considered as per the complications. The nutrition, management of HE, acute variceal bleed and ascites was similar to that for cirrhotics. Acute kidney injury (AKI) was managed initially with terlipressin and albumin, upon failure of which renal replacement therapy was considered with SLED (Sustained Low Efficiency Dialysis). Therapeutic plasmapheresis was done in a few cases with worsening jaundice, coagulopathy in absence of overt sepsis. The patients were declared cured as per the WHO test based definition. Detailed clinical data was collected during hospitalization, at discharge and till a follow up of 28 days.

Severity of COVID-19 This was done based upon the triaging and treatment protocols [21]:

I. Asymptomatic the testing and confirmation was done only for those having contact, travel history but no symptoms.

II. Mild with fever, cough, fatigue, loose motions and other non-specific complaints.

III. Severe with severe pneumonia (i.e. $\mathrm{SpO}_{2}<93 \%$ despite high-flow nasal cannula $\mathrm{O}_{2}$ or respiratory rates $>30$ per minute), features of acute respiratory distress syndrome (ARDS), acute kidney, heart or cir- culatory failure, altered sensorium or combination of above.

Acute liver injury (ALI) Defined as any one of the following (i) Jaundice with a total bilirubin level of $\geq 3 \mathrm{mg} / \mathrm{dl}$, (ii) Acute increase in ALT, AST, SAP, GGT $\geq 2$ times upper normal limit, (iii) PT-INR of $\geq 1.5$ with a previously normal liver parameters.

New onset acute liver injury Defined as those fulfilling the above definition of ALI which developed during hospital stay, but was not there at presentation.

Acute kidney injury increase in serum creatinine levels to 1.5 times of baseline or greater, within 7 days; or absolute value of $\geq 1.5 \mathrm{mg} / \mathrm{dl}$ or urine volume of $<0.5 \mathrm{ml} / \mathrm{kg} / \mathrm{h}$ for $6 \mathrm{~h}$.

Chronic liver disease without cirrhosis Case of chronic hepatitis B or C, MAFLD and Autoimmune Hepatitis diagnosed previously or during current admission with advanced fibrosis, by liver biopsy or transient elastography within last 6 months.

Cirrhosis Patients with clinical features and imaging/ endoscopy suggestive of chronic liver disease and portal hypertension or a previously diagnosed case of cirrhosis.

Acute-on-chronic liver failure (ACLF) Acute hepatic insult manifesting as jaundice and coagulopathy, complicated within 4 weeks by ascites and/or encephalopathy, in a patient with previously diagnosed or undiagnosed chronic liver disease associated with high 4 week mortality [19].

Acute decompensation $(A D)$ Acute development of jaundice (bilirubin $>3 \mathrm{mg} / \mathrm{dl}$ ), large ascites (grade ii-iii), hepatic encephalopathy, gastrointestinal hemorrhage or sepsis, or any combination of these occurring in a period of 90 days before presentation.

Overall complications The presence of AKI with or without need of renal replacement therapy, hypotension or shock, altered sensorium, respiratory distress (as defined in severe ARDS) or need of mechanical ventilation and liver related complications.

Liver related complications Development of complications like worsening of jaundice (bilirubin $>3 \mathrm{mg} / \mathrm{dl}$ ), worsening or development of new ascites, hepatic encephalopathy, acute variceal bleed and spontaneous bacterial peritonitis.

\section{Data collection and statistical analysis}

The retrospectively collected data was analysed as groups i.e. patients having CLD without cirrhosis, those having cirrhosis with or without decompensation, those with or without acute liver injury during COVID-19 infection. Descriptive statistics were expressed as mean $\pm \mathrm{SD}$ or median (IQR). The Student's t-test for continuous data, Fisher's exact test or Pearson's Chi-square test for categorical data and Kaplan-Meier curve with long-rank test was considered for 
survival outcomes. AUROC was used to derive the applicability and cut-off for CTP score to predict mortality. The proportional risk between the groups was calculated as odds ratio. All statistical tests were two-tailed, and a significance level (p) of 0.05 was used. All statistical tests were performed using SPSS.

\section{Results}

We present the data obtained from 13 Asian countries and 62 investigators on the spectrum of liver injury and outcomes in CLD patients infected with SARS-Cov-2. Present study included 408 confirmed COVID-19 cases, of which 175 had no evidence of chronic liver disease and another 5 had inadequate data. (Fig. 1). Altogether, 228 CLD patients were therefore included; 43 (18.9\%) with cirrhosis (including 18 decompensated cirrhosis) and $185(81.1 \%)$ without cirrhosis.

\section{Profile of patients of CLD with or without cirrhosis exposed to SARS-CoV-2 infection}

The patients were mostly in the fifth or sixth decade with high rates of comorbidities. CLD without cirrhosis had male preponderance $(57.8 \%$ versus $41.9 \%, \mathrm{p}=0.01)$ with MAFLD being more common $(61.1 \%$ versus $32.5 \%, \mathrm{p}=0.003)$. The symptoms, laboratory parameters including leukocyte and platelet count and the severity of COVID-19 (18.6\% versus $11.8 \%, \mathrm{p}=0.14$ ) were comparable (Table 1$)$.

More patients of cirrhosis had acute liver injury at admission $(32.6 \%$ vs $20 \%, \mathrm{p}<0.001)$ and also developed new onset liver injury in-hospital $(39.5 \%$ versus $7 \%, \mathrm{p}<0.001)$ who had no ALI at presentation. The ALI occurred in $40 \%$ of those without cirrhosis, but without decompensation. The ALI caused decompensation in $20.7 \%$ of cirrhotics, $9.1 \%$ developed AD and 11.6\% ACLF.

COVID-19 related complications, i.e. acute kidney injury $(18.6 \%$ versus $5.4 \%, \mathrm{p}<0.001)$, respiratory failure $(23.2 \%$ versus $8.6 \%, \mathrm{p}<0.001)$ and hypotension $(14 \%$ versus $3.8 \%$, $\mathrm{p}<0.001$ ) were more common in cirrhotics than CLD without cirrhosis. Those with cirrhosis needed more ICU care

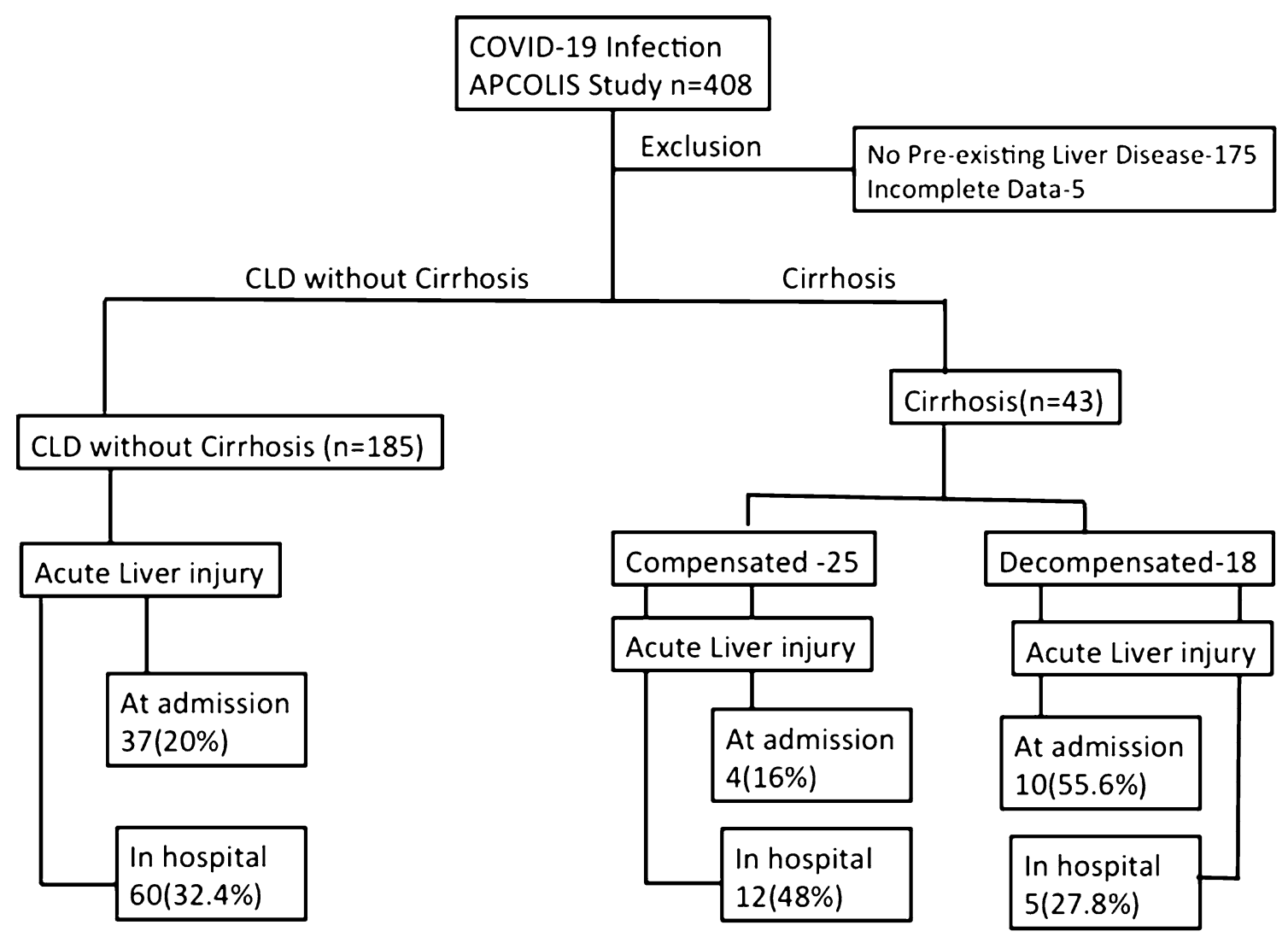

Fig. 1 Flow diagram. Enrolment of patients and acute liver injury. Acute liver injury was seen at presentation in $20 \%$ of CLD without cirrhosis, $16 \%$ of compensated and $55.6 \%$ of decompensated cirrho- sis. Further acute liver injury was noted in $32.4 \%$ of those without cirrhosis and in $48 \%$ of compensated cirrhosis. Where as among the decompensated cirrhotics $27 \%$ had new onset acute liver injury 
Table 1 Baseline parameters among CLD patients with or without cirrhosis exposed to SARS CoV2 infection

\begin{tabular}{|c|c|c|c|}
\hline Parameters & Cirrhosis $(n=43)$ & $\begin{array}{l}\text { CLD-No Cirrhosis } \\
(\mathrm{n}=185)\end{array}$ & $\mathrm{p}$ \\
\hline Age in years $($ mean $\pm S D)$ & $48.3 \pm 15.5$ & $51.8 \pm 14.9$ & 0.15 \\
\hline Gender (Male, n \%) & $25(41.9)$ & $107(57.8)$ & 0.01 \\
\hline Comorbidity (n, \%) & $34(79.1)$ & $150(81.1)$ & 0.83 \\
\hline \multicolumn{4}{|l|}{ Etiology of liver disease } \\
\hline MAFLD (n, \%) & $14(32.5)$ & $113(61.1)$ & 0.003 \\
\hline Viral (n, \%) & $26(60.4)$ & $44(23.8)$ & 0.003 \\
\hline Ethanol (n, \%) & $2(4.6)$ & $13(7.1)$ & 0.45 \\
\hline Others $(\mathrm{n}, \%)$ & $1(2.3)$ & $15(8.2)$ & 0.39 \\
\hline \multicolumn{4}{|l|}{ Type of symptoms } \\
\hline Fever $(\mathrm{n}, \%)$ & $27(62.8)$ & $107(57.8)$ & 0.27 \\
\hline Cough (n, \%) & $29(67.4)$ & $102(55.1)$ & 0.07 \\
\hline Shortness of breath (n, \%) & $8(18.6)$ & $38(20.5)$ & 0.08 \\
\hline \multicolumn{4}{|l|}{ Covid severity } \\
\hline Severe & $8(18.6)$ & $23(11.8)$ & 0.14 \\
\hline \multicolumn{4}{|l|}{ Laboratory parameters } \\
\hline Hemoglobin mg/dl (mean \pm SD) & $13.5 \pm 1.5$ & $13.3 \pm 2.2$ & 0.65 \\
\hline Total WBC10 1 /cc $($ mean $\pm \mathrm{SD})$ & $5.0 \pm 2.1$ & $5.8 \pm 3.1$ & 0.17 \\
\hline Platelet $10^{9} / \mathrm{cc}(\operatorname{mean} \pm \mathrm{SD})$ & $208.6 \pm 100.9$ & $209.8 \pm 91.9$ & 0.95 \\
\hline $\mathrm{T}$ billirubin $\mathrm{mg} / \mathrm{dl}$ (median, range) & $0.9(0.2-17.5)$ & $0.7(0.1-6.4)$ & 0.001 \\
\hline AST IU/L (median, range) & $37(9-4052)$ & $30(11-288)$ & 0.04 \\
\hline ALT IU/L (median, range) & $36(12-1875)$ & $30(6-258)$ & 0.07 \\
\hline SAP IU/L (median, range) & $64(36-181)$ & $67(4-256)$ & 0.86 \\
\hline GGT IU/L (median, range) & $34.5(14-352)$ & $31(4-644)$ & 0.22 \\
\hline S Albumin gm/dl (mean \pm SD) & $3.4 \pm 0.8$ & $3.9 \pm 0.6$ & 0.001 \\
\hline PT-INR $($ mean \pm SD) & $1.6 \pm 2.5$ & $1.2 \pm 1.03$ & 0.18 \\
\hline Creatinine $\mathrm{mg} / \mathrm{ml}$ (median, range) & $0.7(0.3-6.8)$ & $0.5(0.2-9.3)$ & 0.69 \\
\hline \multicolumn{4}{|l|}{ Acute liver injury } \\
\hline At admission (n, \%) & $14(32.6)$ & $37(20)$ & $<0.001$ \\
\hline New onset (n, \%) & $17(39.5)$ & $13(7)$ & 0.01 \\
\hline \multicolumn{4}{|l|}{ Liver injury profile } \\
\hline Worsening decompensation (n, \%) & $4(9.3)$ & 0 & 0.001 \\
\hline $\operatorname{ACLF}(n, \%)$ & $5(11.6)$ & 0 & \\
\hline $\operatorname{ALI}(\mathrm{n}, \%)$ & $16(37.2)$ & $81(43.8)$ & \\
\hline \multicolumn{4}{|l|}{ Treatment received } \\
\hline HCQs + azathioprine (n, \%) & $14(32.5)$ & $48(25.9)$ & 0.19 \\
\hline Antiviral drugs (n, \%) & $12(27.9)$ & $59(31.9)$ & 0.31 \\
\hline Steroid (n, \%) & $6(13.9)$ & $11(5.9)$ & 0.03 \\
\hline IVIG (n, \%) & $3(6.9)$ & $5(2.7)$ & 0.11 \\
\hline Therapeutic plasma exchange (n, \%) & $3(6.9)$ & $2(1.1)$ & 0.03 \\
\hline Convalescent Plasma (n, \%) & $2(4.6)$ & $2(1.1)$ & 0.09 \\
\hline \multicolumn{4}{|l|}{ COVID-19 related complications } \\
\hline Respiratory Failure (n, \%) & $10(23.2)$ & $16(8.6)$ & $<0.001$ \\
\hline Kidney Failure (n, \%) & $8(18.6)$ & $10(5.4)$ & $<0.001$ \\
\hline Shock $(\mathrm{n}, \%)$ & $6(14)$ & $7(3.8)$ & $<0.001$ \\
\hline \multicolumn{4}{|l|}{ Disease course } \\
\hline Need of ICU care (n, \%) & $11(25.6)$ & $23(12.4)$ & $<0.001$ \\
\hline Liver related complications (n, \%) & $14(32.6)$ & $26(14.1)$ & 0.007 \\
\hline Mortality (n, \%) & $7(16.3)$ & $5(2.7)$ & 0.002 \\
\hline Hospital stay (median in days) & $19(2-28)$ & $19(2-28)$ & 0.98 \\
\hline
\end{tabular}


( $25.6 \%$ versus $12.4 \%, \mathrm{p}<0.001)$, developed higher liver related complications $(32.6 \%$ versus $14.1, \mathrm{p}=0.007)$ leading to higher mortality $(16.3 \%$ versus $2.7 \%, \mathrm{p}=0.002)$.

\section{Profile of Cirrhosis with or without decompensation exposed to SARS-CoV-2 infection}

The present study had 43 cirrhosis patients; 18 (41.8\%) with prior decompensation, 16 (37.2\%) with Child B and 3 (9\%) with Child C. Most common etiology was viral $(26,60.5 \%)$, followed by MAFLD $(14,32.6 \%)$, alcohol $(2,4.7 \%)$ and autoimmune hepatitis $(1,2.3 \%)$ [Table S1].

Severe COVID-19 was more common among decompensated $[33.3 \%$ versus $8 \%, \mathrm{OR}=5.5(1.1-44.3), \mathrm{p}=0.02]$ with higher complications, i.e. acute kidney injury $(33.3 \%$ versus $8 \%, \mathrm{p}=0.02)$, respiratory failure $(50 \%$ versus $4 \%, \mathrm{p}<0.001)$ and circulatory failure $(27.8 \%$ versus $4 \%, \mathrm{p}=0.02)$. Decompensated cirrhotics had more liver injury $[71.4 \%$ versus $13.8 \%, \mathrm{OR}=6.2(1.55-29.13), \mathrm{p}<0.001]$ at presentation. The liver related [ $44.4 \%$ versus $24 \%, \mathrm{OR}=3.24(0.88-12.5)$, $\mathrm{p}=0.08]$ and overall complications were more in decompensated cirrhosis with greater need for ICU care and higher mortality [OR $=11.3(1.5-288.1), \mathrm{p}=0.008]$.

\section{SARS-CoV-2 infection related liver injury and outcome in CLD without cirrhosis}

The liver injury in CLD patients without cirrhosis was noted more in those with severe COVID-19 [18\% versus 5.7\%, $\mathrm{OR}=3.76(1.38-11.8), \mathrm{p}=0.004]$. Patients of CLD with diabetes had higher risk $[57.7 \%$ versus $39.7 \%, p=0.01$, $\mathrm{OR}=2.061 .14-3.73)]$ of liver injury. Patients with liver injury, needed more ICU admissions $(20.6 \%$ versus $3.4 \%$, $\mathrm{p}<0.001)$ with higher liver related $(24.7 \%$ versus $2.3 \%$, $\mathrm{p}<0.001)$ and overall complications $[39.2 \%$ versus $6.8 \%$, $\mathrm{p}<0.001)$. However, the recovery, hospital stay and associated mortality were comparable among those with or without liver injury (Table 2).

\section{SARS-CoV-2 infection related liver injury and outcome in cirrhosis}

The acute liver injury was seen in $14(32.6 \%)$ patients (Table 3). The age, gender or presence of diabetes were comparable, but the risk of liver injury was more with diabetes (64.3\% versus $17.2 \%, p=0.002)$. Those with liver injury had higher CTP, MELD score and were often decompensated $(55.6 \%$ versus $8 \%, \mathrm{p}<0.001)$ and had also contracted more severe COVID-19 disease [ $42.8 \%$ versus $6.9 \%, \mathrm{OR}=9.5$ (1.7-79.5)]. The COVID-19 related complications, such as renal, respiratory or circulatory failure were higher among cirrhotics with liver injury and required more ICU
Table 2 Profile of CLD without cirrhosis and liver injury with COVID-19

\begin{tabular}{|c|c|c|c|}
\hline Parameters & $\begin{array}{l}\text { Acute liver injury } \\
(\mathrm{n}=97)\end{array}$ & $\begin{array}{l}\text { No acute liver injury } \\
(\mathrm{n}=88)\end{array}$ & $\mathrm{p}$ \\
\hline Age in years $($ mean $\pm S D)$ & $51.7 \pm 14.7$ & $51.8 \pm 15.4$ & 0.97 \\
\hline Gender (n, \%) & $58(59.8)$ & $49(55.7)$ & 0.65 \\
\hline \multicolumn{4}{|l|}{ Presence of comorbidity } \\
\hline Diabetes Mellitus (n, \%) & $56(57.7)$ & $35(39.7)$ & 0.01 \\
\hline Obesity (n, \%) & $36(37.1)$ & $46(52.3)$ & 0.02 \\
\hline Cardiovascular (n, \%) & $20(20.6)$ & $27(30.7)$ & 0.06 \\
\hline \multicolumn{4}{|l|}{ Etiology of liver disease } \\
\hline MAFLD (n, \%) & $48(55.2)$ & $55(69.6)$ & 0.03 \\
\hline Viral Hepatitis (n, \%) & $25(28.7)$ & $15(19)$ & 0.07 \\
\hline Ethanol (n, \%) & $7(8)$ & $5(6.3)$ & 0.34 \\
\hline \multicolumn{4}{|l|}{ COVID-19 related complications } \\
\hline Respiratory Failure (n, \%) & $13(13.4)$ & $3(3.4)$ & 0.01 \\
\hline Kidney failure (n, \%) & $8(8.2)$ & $2(2.3)$ & 0.04 \\
\hline Circulatory failure (n, \%) & $6(6.2)$ & $1(1.1)$ & 0.04 \\
\hline \multicolumn{4}{|l|}{ Disease course } \\
\hline Need of ICU care (n, \%) & $20(20.6)$ & $3(3.4)$ & $<0.001$ \\
\hline Liver related complication (n, \%) & $24(24.7)$ & $2(2.3)$ & $<0.001$ \\
\hline Over all complication (n, \%) & $38(39.2)$ & $6(6.8)$ & $<0.001$ \\
\hline Death $(\mathrm{n}, \%)$ & $3(2.1)$ & $2(2.3)$ & 1.00 \\
\hline Hospital stay (median in days) & $20(2-28)$ & $17(2-28)$ & 0.07 \\
\hline
\end{tabular}


Table 3 Profile of liver injury due to COVID-19 among cirrhotics

\begin{tabular}{|c|c|c|c|}
\hline Parameters & $\begin{array}{l}\text { Acute liver injury at } \\
\text { admission }(n=14)\end{array}$ & $\begin{array}{l}\text { No acute liver injury at } \\
\text { admission }(n=29)\end{array}$ & $\mathrm{p}$ \\
\hline Age in years $($ mean $\pm S D)$ & $43.9 \pm 13.8$ & $50.1 \pm 16.1$ & 0.23 \\
\hline Gender (Male, n, \%) & $6(42.8)$ & $19(65.5)$ & 0.21 \\
\hline \multicolumn{4}{|l|}{ Comorbidities } \\
\hline Diabetes mellitus (n, \%) & $6(42.9)$ & $14(48.3)$ & 0.49 \\
\hline Obesity (n, \%) & $9(64.3)$ & $5(17.2)$ & 0.002 \\
\hline Cardiovascular (n, \%) & $3(21.4)$ & $7(24.1)$ & 0.84 \\
\hline \multicolumn{4}{|l|}{ COVID disease Severity } \\
\hline Severe $(\mathrm{n}, \%)$ & $6(42.8)$ & $2(6.9)$ & 0.01 \\
\hline \multicolumn{4}{|l|}{ Laboratory parameters } \\
\hline Hemoglobin $($ mean \pm SD) & $14.1 \pm 1.04$ & $13.2 \pm 1.63$ & 0.10 \\
\hline Total WBC $($ mean \pm SD $)$ & $6.18 \pm 2.04$ & $4.48 \pm 1.96$ & 0.02 \\
\hline Absolute lymphocyte (mean \pm SD) & $1.61 \pm 0.89$ & $0.99 \pm 0.67$ & 0.07 \\
\hline Platelet (mean \pm SD) & $176.8 \pm 81.7$ & $222.6 \pm 106.7$ & 0.21 \\
\hline $\mathrm{T}$ billirubin $\mathrm{mg} / \mathrm{dl}$ (median, range) & $1.4(0.9-17.5)$ & $0.6(0.2-2.8)$ & 0.04 \\
\hline AST IU/L (median, range) & $52(17-4061)$ & $31(9-66)$ & 0.01 \\
\hline ALT IU/L (median, range) & $42(14-1875)$ & $35(12-76)$ & 0.03 \\
\hline SAP IU/L (median, range) & $78.5(36-181)$ & $64(44-121)$ & 0.07 \\
\hline GGT IU/L (median, range) & $61(14-352)$ & $38(15-108)$ & 0.01 \\
\hline $\mathrm{S}$ albumin (median, range) & $2.89 \pm 0.78$ & $3.92 \pm 0.29$ & 0.001 \\
\hline PT-INR (median, range) & $1.31 \pm 1.17$ & $0.64 \pm 0.47$ & 0.02 \\
\hline Creatinine (median, range) & $1(0.3-7.1)$ & $0.6(0.2-4.3)$ & 0.18 \\
\hline \multicolumn{4}{|l|}{ Cirrhosis disease severity } \\
\hline Decompensation at presentation (n, \%) & $10(71.4)$ & $4(13.8)$ & $<0.001$ \\
\hline CTP score $($ mean $\pm \mathrm{SD})$ & $8.1 \pm 2.1$ & $6.0 \pm 1.05$ & 0.004 \\
\hline \multicolumn{4}{|l|}{ Child Pugh class } \\
\hline $\mathrm{A}(\mathrm{n}, \%)$ & $5(35.7)$ & $19(65.5)$ & 0.02 \\
\hline $\mathrm{B}(\mathrm{n}, \%)$ & $6(42.9)$ & $10(34.5)$ & \\
\hline $\mathrm{C}(\mathrm{n}, \%)$ & $3(21.4)$ & 0 & \\
\hline MELD Score (mean \pm SD) & $20.5 \pm 9.8$ & $12.3 \pm 6.4$ & 0.02 \\
\hline CTP score at discharge $($ mean $\pm \mathrm{SD})$ & $9.29 \pm 1.98$ & $7.41 \pm 1.32$ & 0.005 \\
\hline \multicolumn{4}{|l|}{ Liver injury profile } \\
\hline Acute decompensation & $4(28.6)$ & 0 & $<0.001$ \\
\hline Acute on chronic liver failure & $4(28.6)$ & $1(3.4)$ & \\
\hline Acute liver injury & $4(28.6)$ & $12(41.4)$ & \\
\hline Not affected & $2(14.3)$ & $16(55.2)$ & \\
\hline \multicolumn{4}{|l|}{ COVID-19 related complications } \\
\hline Respiratory failure (n, \%) & $6(42.8)$ & $3(10.3)$ & 0.04 \\
\hline Kidney failure (n, \%) & $5(35.7)$ & $1(3.4)$ & 0.03 \\
\hline Shock (n, \%) & $6(42.8)$ & $2(6.8)$ & 0.04 \\
\hline \multicolumn{4}{|l|}{ Liver related complications } \\
\hline Worsening jaundice (n, \%) & $5(35.7)$ & $5(17.2)$ & 0.02 \\
\hline Worsening ascites (n, \%) & $5(35.7)$ & $5(17.2)$ & 0.02 \\
\hline Hepatic encephalopathy (n, \%) & $3(21.4)$ & 0 & 0.03 \\
\hline Acute variceal bleed (n, \%) & $3(21.4)$ & $1(3.4)$ & 0.05 \\
\hline Spontaneous bacterial peritonitis (n, \%) & $3(21.4)$ & 0 & 0.03 \\
\hline \multicolumn{4}{|l|}{ Disease course } \\
\hline Need of ICU care (n, \%) & $66(42.8)$ & $1(3.4)$ & 0.001 \\
\hline Liver related complications (n, \%) & $8(57.1)$ & $6(20.7)$ & 0.02 \\
\hline Over all complications (n, \%) & $8(57.1)$ & $6(20.7)$ & 0.02 \\
\hline Death (n, \%) & $6(42.9)$ & $2(6.9)$ & 0.03 \\
\hline
\end{tabular}


Table 3 (continued)

\begin{tabular}{llll}
\hline Parameters & $\begin{array}{l}\text { Acute liver injury at } \\
\text { admission }(\mathrm{n}=14)\end{array}$ & $\begin{array}{l}\text { No acute liver injury at } \\
\text { admission }(\mathrm{n}=29)\end{array}$ & $\mathrm{p}$ \\
\hline Hospital stay (median in days) & $20(3-28)$ & $16(2-28)$ & 0.46 \\
\hline
\end{tabular}

care $(42.8 \%$ versus $3.4 \%, \mathrm{p}=0.001)$ with higher mortality (42.8\% versus $6.9 \%, \mathrm{p} 0.03)$. The liver related complications, i.e. worsening of jaundice, ascites, hepatic encephalopathy, variceal bleed and SBP happened more with COVID-19 related liver injury irrespective of decompensation.

\section{Degree of liver injury over time and predictors of mortality}

The liver injury may be evident at presentation or develop and progress during the course of infection. The AST/ALT ratio, total bilirubin and $\mathrm{R}$ value (ALT/ALP ratio) were helpful in predicting survival in cirrhotics (Fig. S1). The nonsurvivors had higher AST, and an AST/ALT ratio of $>1.4$ [AUROC 0.95, HR $=1.4$ (95 CI 2.5-5.4), $\mathrm{p}=0.02$ ] predicted mortality among cirrhotics. The mean total bilirubin remained elevated to $>9 \mathrm{mg} / \mathrm{dl}$ till death in non-survivors. They also had a low $\mathrm{R}$ value $[\mathrm{p}=0.02]$. The liver injury occurred more towards the end of second week or early third week in non-cirrhotics (Fig. S2), but was evident at presentation or developed in the first week in cirrhotic patients.

The mortality was comparable among non-cirrhotics and compensated cirrhosis, despite more complications, liver injury and liver related complications in the later. This underscores the concept of adequacy of available hepatic reserve for recovery. It was further substantiated by the fact that decompensated cirrhosis had nearly twice the mortality seen in compensated cirrhosis [ $33 \%$ vs. $16.3 \%, \mathrm{OR}=2.5$ (95 CI 0.7-9.4) $\mathrm{p}=0.05]$. The CTP score at presentation can predict survival in a cirrhosis [AUROC 0.94 , sensitivity $86 \%$ and specificity of 94\%] and a score above 8 showed high mortality $(85.7 \%, \mathrm{HR}=19.2$ (95 CI 2.3-163.3), p < 0.001) (Fig. 2).

\section{Discussion}

The results of this large multinational study, including a cohort of 228 patients, SARS-CoV-2 infection produces acute liver injury in $43 \%$ of CLD patients without cirrhosis. Additionally, 20\% of compensated cirrhosis patients develop either ACLF or acute decompensation. Liver related complications were seen in nearly half of the decompensated cirrhotics, which were of greater severity and with higher mortality.

The median age of presentation was sixth decade, but age above 60 years was not a poor prognostic factor in presence of liver disease. Comorbidities like MAFLD, obesity and diabetes were present in nearly $80 \%$ of the patients. Presence of obesity is known to increase the risk of liver injury [22]. We observed that obese cirrhotics had more acute liver injury than normal weight patients [OR $8.9(95 \mathrm{CI}=1.9-38.8) \mathrm{p}=0.02]$, with higher complication rates. Also, CLD patients with diabetes had more acute liver injury [OR $2.1(95 \mathrm{CI}=1.1-3.73), \mathrm{p}=0.01]$. Higher incidence of ALI has also been reported in diabetics without liver disease [23]. In the present study, MAFLD was the commonest cause for CLD without cirrhosis, whereas hepatitis B was common in cirrhotics [24]. Interestingly, alcohol was found to be a less frequent cause of CLD in Asian patients infected with SARS-Co-V2.

SARS-Co-V2 infection related injury is systemic and can be more common and severe in immunocompromised individuals [14]. Presence of CAIDS can predispose CLD patients to severe infection [18]. The acute liver injury was more common and severe in patients with decompensated cirrhosis, indicating correlation between the degree of CAIDS and synthetic functions of liver. Liver injury in COVID-19 is multifactorial. It could be due to the hypoxic injury in patients with severe pneumonia [25] or intense cytokine storm with severe infection [26], or due to the drugs used as therapeutic agents [27].

The pattern of liver injury in the CLD patients was suggestive of a dominant hepatocellular injury [28] as serum ALT levels were higher than the AST levels among noncirrhotics. Interestingly, the liver injury pattern was different in cirrhotic patients. A rapid and early worsening of jaundice and higher AST than ALT and low R value was seen in cirrhotic patients, more so in those who did not survive. This indicates that liver injury in cirrhosis is either drug induced or hypoxia related [12, 27, 29]. There was only an insignificant rise in serum alkaline phosphatase and gamma glutamyl transferase, indicating limited virus related injury to liver due to the over expression of ACE2 on cholangiocytes. However, more histological and experimental studies are required to ascertain this.

While mild derangements in the liver functions are expected in systemic infections, the term ALI is used to differentiate more severe liver injury. We defined ALI with serum bilirubin of $3 \mathrm{mg} / \mathrm{dl}$ or above and liver enzymes raised at least 2 times upper limit of normal. Similar criteria for ALI were used in COVID-19 earlier [5, 27]. Our study supports the concept of ALI even in patients with CLD and compensated cirrhosis. However, its relevance for development of complications and mortality would require a larger cohort of patients with different etiologies. 

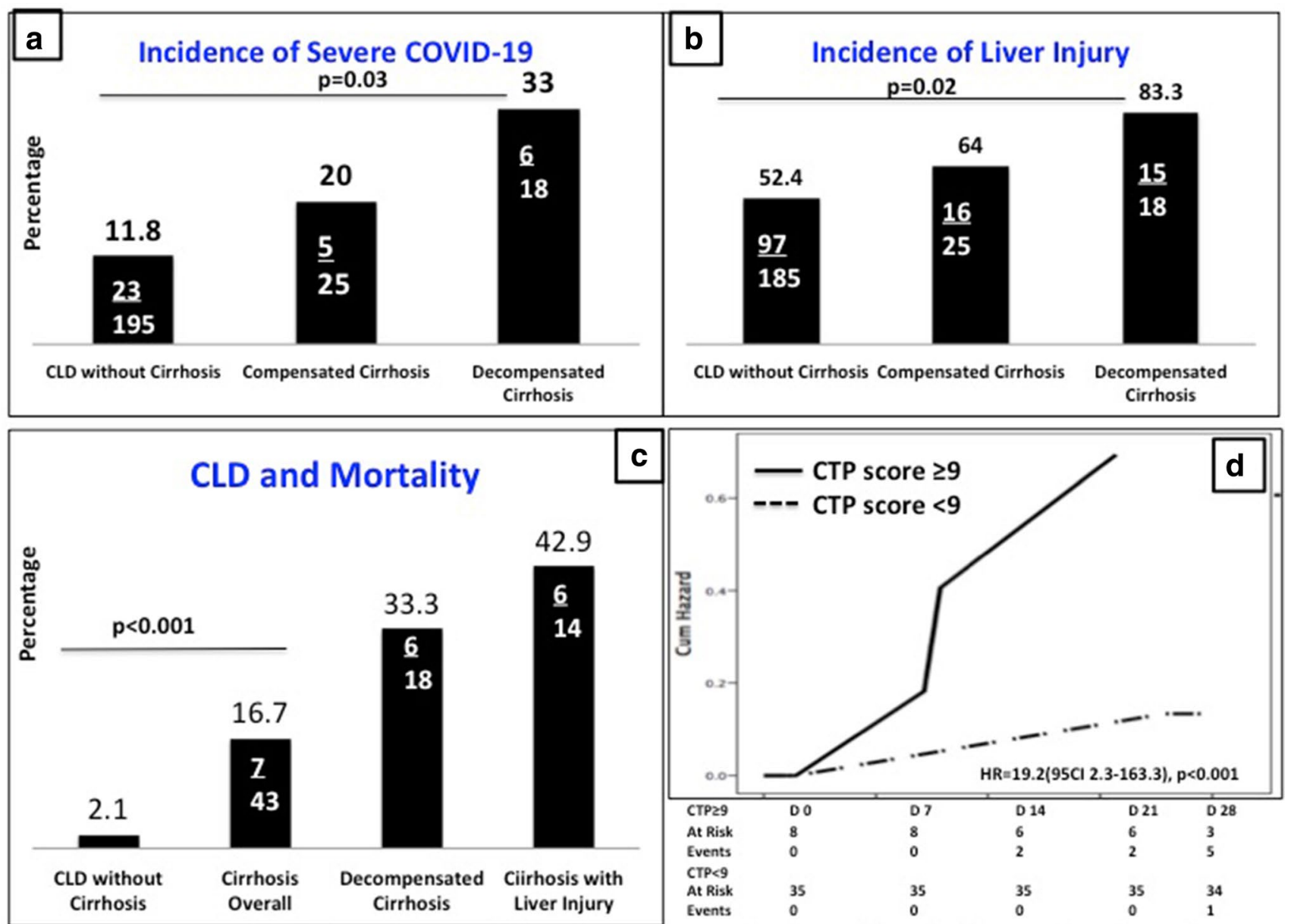

Fig. 2 COVID-19 and Spectrum of CLD. a The incidence of severe disease due to COVID-19 increases progressively among non-cirrhotics to compensated to decompensated cirrhosis $(p=0.03)$ as the synthetic function decreased. b There is similar trend for acute liver injury $(p=0.02)$. $\mathbf{c}$ The mortality increased with SARS CoV2 infection significantly among cirrhotics than those without cirrhosis

A correlation between hepatic reserve and liver synthetic functions was observed in development of liver related complications from COVID-19. The liver injury occurred towards the third week in CLD patients without cirrhosis, but developed within the first week in cirrhotics. The late trend of injury in CLD without cirrhosis has also been reported recently [28]. The fact that cirrhosis patients may have a more serious injury, one should be careful in the choice of antiviral agents. We could not ascertain the role of antivirals, azithromycin and hydroxycholorquine, used in nearly one third of the patients, due to multiple mechanisms of liver injuries in cirrhotics.

Significant liver failure in the form of ACLF or acute decompensation was seen in $20 \%$ of the cirrhotic patients. This observation adds a new dimension to the existing literature on COVID-19. The data indicates that non-hepatotropic infections, such as SARS CoV2, can directly precipitate a $(\mathrm{p}<0.001)$ and with decompensation. The mortality is highest $(43 \%)$ in the spectrum with onset of liver injury. d Among cirrhosis those exposed to SARS CoV2 infection, the outcome is poor with CTP score 9 or more [AUROC 0.94 , sensitivity $86 \%$ and specificity of $94 \%, \mathrm{HR}=19.2$ (95 CI 2.3-163.3), $\mathrm{p}<0.001]$

hepatic injury severe enough to cause liver failure in cirrhotic patients [30].

The present study has the limitation of non-availability of histological data for identifying those with mild or advanced fibrosis. The COVID-19 related complications leading to liver related complications is not well known in absence of biopsy, however in the era of pandemic and the recent worsening with SARS CoV2 infection suggest a temporal association. However, this was not possible in the current state of the spreading pandemic, especially in the absence of an effective antiviral or hepato-protective agent.

In summary, pre-existing liver disease is an added risk in severe COVID-19. Liver related complications, overall complications and outcomes correlate with the existing hepatic reserve. Acute liver injury is more severe and progressive in patients with decompensated cirrhosis and is associated with high mortality. Comorbidities like diabetes, obesity 
and MAFLD do aggravate the risk of liver injury. Hence, patients with COVID-19 disease having chronic liver disease should be properly attended to, prioritized in management and duly prognosticated. These facts assume immediate relevance as at present, liver transplant services in most countries are either on hold or are available only for acute liver failure patients.

Author contributions SKS and AC conceptualised the research design, GL has given the critical input for designing the manuscript, AC prepared the manuscript along with the statistical analysis, rest of the authors contributed the data and suggested modification, SKS did the final proof reading and provided all the logistic support.

Funding None.

\section{Compliance with ethical standards}

Conflict of interest The authors declare no conflicts of interest.

\section{References}

1. Coronavirus disease (COVID-19) Situation Report - 113. Data as received by WHO from national authorities. 2020. https://www. who.int/docs/default-source/coronaviruse/situation-reports/20200 512-covid-19-sitrep-113.pdf?sfvrsn=feac3b6d_2.

2. Lu R, Zhao X, Li J, Niu P, Yang B, Wu H, et al. Genomic characterisation and epidemiology of 2019 novel coronavirus: implications for virus origins and receptor binding. Lancet 2020;395(10224):565-574

3. Guan WJ, Ni ZY, Hu Y, Liang WH, Ou CQ, He JX, et al. Clinical characteristics of coronavirus disease 2019 in China. N Engl J Med. 2020. https://doi.org/10.1056/NEJMoa2002032

4. Yang X, Yu Y, Xu J, Shu H, Xia J, Liu H, et al. Clinical course and outcomes of critically ill patients with SARS-CoV-2 pneumonia in Wuhan, China: a single-centered, retrospective, observational study. Lancet Respir Med. 2020. https://doi.org/10.1016/s2213 -2600(20)30079-5

5. Chen C, Zhang XR, Ju ZY, He WF. Advances in the research of cytokine storm mechanism induced by Corona Virus Disease 2019 and the corresponding immunotherapies. Zhonghua Shaoshang Zazhi 2020;36:E005

6. Liu Z, Xiao X, Wei X, Li J, Yang J, Tan H, et al. Composition and divergence of coronavirus spike proteins and host ACE2 receptors predict potential intermediate hosts of SARS-CoV-2. J Med Virol. 2020. https://doi.org/10.1002/jmv.25726 (epub ahead of print)

7. Xu H, Zhong L, Deng J, et al. High expression of ACE2 receptor of 2019-nCoV on the epithelial cells of oral mucosa. Int J Oral Sci. 2020. https://doi.org/10.1038/s41368-020-0074-x

8. Xie H, Zhao J, Lian N, et al. Clinical characteristics of non-ICU hospitalized patients with coronavirus disease 2019 and liver injury: a retrospective study. Liver Int. 2020 (online ahead of print)

9. Zhang C, Shi L, Wang FS. Liver injury in COVID-19: management and challenges. Lancet Gastroenterol Hepatol. 2020. https ://doi.org/10.1016/S2468-1253(20)30057-1

10. Fan Z, Chen L, Li J, et al. Clinical features of COVID-19-related liver damage. medRxiv. https://doi.org/10.1101/2020.02.26.20026 971
11. Li J, Jian-Gao F. Characteristics and mechanism of liver injury in 2019 coronavirus disease. J Clin Transl Hepatol. 2020. https:// doi.org/10.14218/JCTH.2020.00019

12. Feng G, Zheng KI, Yan Q-Q, et al. COVID-19 and liver dysfunction: current insights and emergent therapeutic strategies. J Clin Transl Hepatol 2020;8(1):18-24

13. Tian S, Xiong Y, Liu H, et al. Pathological study of the 2019 novel coronavirus disease (COVID-19) through postmortem core biopsies. Mod Pathol. 2020. https://doi.org/10.1038/s4137 9-020-0536-x

14. D'Antiga L. Coronaviruses and immunosuppressed patients. The facts during the third epidemic. Liver Transpl. 2020 (online ahead of print)

15. Sarin SK. Fast, faster, and fastest: science on the run during COVID-19 drama-do not forget the liver. Hepatol Int. 2020 https://doi.org/10.1007/s12072-020-10042-0

16. Eslam M, Sanyal AJ, George J, on behalf of the International Consensus Panel, et al. MAFLD: a consensus-driven proposed nomenclature for metabolic associated fatty liver disease. Gastroenterology 2020;158(7):1999-2014.e1. https://doi.org/10.1053/j. gastro.2019.11.312

17. Yang AP, Liu J, Tao W, Li HM. The diagnostic and predictive role of NLR, d-NLR and PLR in COVID-19 patients. Int Immunopharmacol. 2020. https://doi.org/10.1016/j.intimp.2020.106504

18. Albillos A, Lario M, Álvarez-Mon M. Cirrhosis-associated immune dysfunction: distinctive features and clinical relevance. J Hepatol 2014;61(6):1385-1396. https://doi.org/10.1016/j. jhep.2014.08.010

19. Sarin SK, Choudhury A. Acute-on-chronic liver failure. Curr Gastroenterol Rep 2016;18(12):61 PMID: 27747458

20. Laboratory testing for 2019 novel coronavirus $(2019-n C o V)$ in suspected human cases. WHO Interim guidance. COVID-19: Laboratory and diagnosis. 2020. https://www.who.int/publicatio ns/i/item/laboratory-testing-for-2019-novel-coronavirus-in-suspe cted-human-cases-20200117.

21. Clinical management of COVID-19. WHO interim guidance. COVID-19: Clinical care. 2020. https://www.who.int/publicatio ns/i/item/clinical-management-of-covid-19.

22. Stefan N, Birkenfeld AL, Schulze MB, et al. Obesity and impaired metabolic health in patients with COVID-19. Nat Rev Endocrinol. 2020. https://doi.org/10.1038/s41574-020-0364-6

23. Kumar A, Arora A, Sharma P, et al. Is diabetes mellitus associated with mortality and severity of COVID-19? A meta-analysis. Diabetes Metab Syndr Clin Res Rev 2020;14:535-545

24. Ji D, Qin E, Xu J, Zhang D, Cheng G, Wang Y, Lau G. Implication of non- alcoholic fatty liver diseases (NAFLD) in patients with COVID-19: a preliminary analysis. J Hepatol. 2020. https://doi. org/10.1016/j.jhep.2020.03.044

25. Zhang Y, Zheng L, Liu L, et al. Liver impairment in COVID-19 patients: a retrospective analysis of 115 cases from a single center in Wuhan City, China. Liver Int. 2020 (online ahead of print)

26. Ye Q, Wang B, Mao J. The pathogenesis and treatment of the 'cytokine storm' in COVID-19. J Infect. 2020. https://doi. org/10.1016/j.jinf.2020.03.037

27. Cai Q, Huang D, Yu H, et al. COVID-19: abnormal liver function tests. J Hepatol. 2020. https://doi.org/10.1016/j.jhep.2020.04.006

28. Lei F, Liu YM, Zhou F, Qin JJ, Zhang P. Longitudinal association between markers of liver injury and mortality in COVID-19 in China. Hepatology. 2020. https://doi.org/10.1002/hep.31301

29. Kullak-Ublick GA, Andrade RJ, Merz M, et al. Drug-induced liver injury: recent advances in diagnosis and risk assessment. Gut 2017;66:1154-1164

30. Sarin SK, Choudhury A, Sharma MK, APASL ACLF Research Consortium (AARC) for APASL ACLF working Party, et al. Acute-on-chronic liver failure: consensus recommendations of 
the Asian Pacific association for the study of the liver (APASL): an update. Hepatol Int 2019;13(4):353-390
Publisher's Note Springer Nature remains neutral with regard to jurisdictional claims in published maps and institutional affiliations.

\section{Affiliations}

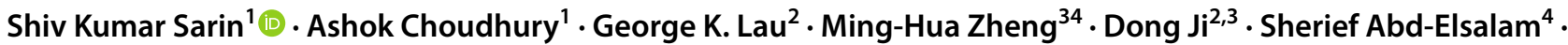
Jaeseok Hwang ${ }^{5}$. Xiaolong Qi ${ }^{6}$. lan Homer Cua ${ }^{7}$. Jeong III Suh ${ }^{8}$. Jun Gi Park ${ }^{8}$. Opass Putcharoen ${ }^{9}$. Apichat Kaewdech ${ }^{10}$. Teerha Piratvisuth $^{10}$. Sombat Treeprasertsuk ${ }^{9}$. Sooyoung Park ${ }^{11}$. Salisa Wejnaruemarn ${ }^{9}$. Diana A. Payawal ${ }^{12}$. Oidov Baatarkhuu ${ }^{13}$. Sang Hoon $\mathrm{Ahn}^{14}$. Chang Dong Yeo ${ }^{14}$. Uzziel Romar Alonzo ${ }^{12}$. Tserendorj Chinbayar ${ }^{15} \cdot$ Imelda M. Loho $^{16}$. Osamu Yokosuka ${ }^{17}$. Wasim Jafri ${ }^{18}$. Soeksiam Tan $^{19} \cdot$ Lau Ing Soo $^{19}$. Tawesak Tanwande $^{20}$. Rino Gani ${ }^{21}$ • Lovkesh Anand ${ }^{22}$. Eslam Saber Esmail ${ }^{4}$. Mai Khalaf ${ }^{4}$. Shahinul Alam ${ }^{23}$. Chun-Yu Lin ${ }^{24} \cdot$ Wan-Long Chuang ${ }^{24}$ - A. S. Soin ${ }^{25} \cdot$ Hitendra K. Garg ${ }^{26} \cdot K_{\text {Kemal Kalista }}^{27} \cdot$ Badamnachin Batsukh $^{13}$. Hery Djagat Purnomo ${ }^{28}$. Vijay Pal Dara ${ }^{29}$. Pravin Rathi ${ }^{30} \cdot$ Mamun Al Mahtab $^{23}$. Akash Shukla ${ }^{31} \cdot$ Manoj K. Sharma $^{1}$. Masao Omata ${ }^{32,33}$. APASL COVID Task Force, APASL COVID Liver Injury Spectrum Study (APCOLIS Study-NCT 04345640)

1 Department of Hepatology and Liver Transplant, Institute of Liver and Biliary Sciences, New Delhi 110070, India

2 Humanity and Health Clinical Trial Center, Hong Kong SAR, China

3 Fuyang Second People's Hospital, Fuyang, China

4 Tropical Medicine and Infectious Diseases Department, Tanta University, Tanta, Egypt

5 Keimyung University Dongsan Hospital, Daegu, South Korea

6 CHESS Center, Institute of Portal Hypertension, The First Hospital of Lanzhou University, Lanzhou, China

7 Institute of Digestive and Liver Diseases, St. Luke's Medical Center, Global City, Philippines

8 Department of Internal Medicine, Dongguk University Gyeongju Hospital, Gyeongju, South Korea

9 Division of Gastroenterology, Department of Medicine, Faculty of Medicine, Chulalongkorn University and Thai Red Cross, Bangkok, Thailand

10 Gastroenterology and Hepatology Unit, Department of Medicine, Prince of Songkla University, Songkhla, Thailand

11 Kyungpook National University Hospital, Daegu, South Korea

12 Department of Internal Medicine, Fatima University Medical Center, Valenzuela, Philippines

13 Department of Infectious Diseases, School of Medicine, Mongolian National University of Medical Sciences, Ulan Bator, Mongolia

14 Severance Hospital, Yonsei University College of Medicine, Seoul, South Korea

15 National Center for Communicable Diseases, Ulan Bator, Mongolia

16 Department of Gastroenterology and Hepatology "Dharmais", National Cancer Hospital, Jakarta, Indonesia

17 Chiba University, Chiba, Japan
18 Department of Medicine, WGO Training Center, Aga Khan University, Karachi, Pakistan

19 Department of Hepatology, Selayang Hospital, Batu Caves, Malaysia

20 Division of Gastroenterology, Department of Medicine, Faculty of Siriraj Hospital, Mahidol University, Bangkok, Thailand

21 Division of Hepatobiliary, Cipto Mangunkusuamo Hospital, University of Indonesia, Jakarta, Indonesia

22 Hepatologist, Manipal Hospital, New Delhi, India

23 Department of Hepatology, Bangabandhu Sheikh Mujib Medical University, Dhaka, Bangladesh

24 Division of Infectious Diseases, School of Medicine, Kaohsiung Medical University, Kaohsiung Medical University Hospital, Kaohsiung, Taiwan

25 Liver Transplant Surgery, Medanta, The Medicity, Gurugram, Haryana, India

26 Hepatologist and Gastroenterologist, Indraprastha Apollo Hospital, New Delhi, India

27 Faculty of Medicine, Cipto Mangunkusumo Hospitall, Universitas, Jakarta, Indonesia

28 Kariadi Hospital, Diponegoro University, Semarang, Indonesia

29 Yerevan Medical University, Yerevan, Armenia

30 Department of Gastroenterology, T.N. Medical College, B.Y.L. Nair. Ch. Hospital, Mumbai, India

31 Department of Gastroenterology, Seth GSMC and KEM Hospital, Mumbai, India

32 Department of Gastroenterology, Yamanashi Prefectural Central Hospital, Kofu, Yamanashi, Japan

33 The University of Tokyo, Tokyo, Japan

34 Department of Hepatology, NAFLD Research Center, The First Affiliated Hospital of Wenzhou Medical University, Wenzhou, China 